\title{
How Can Galectin-3 as a Biomarker of Fibrosis Improve Atrial Fibrillation Diagnosis and Prognosis?
}

\author{
Maria Mariana Barros Melo da Silveira ${ }^{\mathrm{a}, \mathrm{b}, \mathrm{f}}$, Joao Victor Batista Cabral ${ }^{\mathrm{a}}$, Bruno de Melo Souza ${ }^{\mathrm{c}}$, \\ Louis Hussein Patu Hazime ${ }^{\mathrm{c}}$, Sara Larissa de Melo Araujo ${ }^{\mathrm{d}}$, Amanda Tavares Xavierc, e, \\ Edivaldo Bezerra Mendes Filho ${ }^{a}$, Luydson Richardson da Silva Vasconcelose, \\ Dario Celestino Sobral Filho ${ }^{\mathrm{b}, \mathrm{c}}$, Dinaldo Cavalcanti de Oliveira ${ }^{\mathrm{a}, \mathrm{b}}$
}

\begin{abstract}
Galectin-3 (Gal-3) is a biomarker of fibrosis that has been associated with atrial remodeling. Acknowledging the presence of a biomarker in patients with atrial fibrillation (AF) can allow for a better clinical treatment. The aim of this study was to assess the association of Gal-3 with atrial fibrosis in patients with AF. This is a systematic review study. From the total number of studies analyzed, 12 demonstrated a relation between atrial fibrosis and Gal-3 in patients with AF and presented statistically significant association values. We conclude that Gal-3 is associated with atrial fibrosis in patients with AF in all types, as well as after the arrhythmia treatment by ablation.
\end{abstract}

Keywords: Galectin-3; Atrial fibrosis; Atrial fibrillation; Clinical studies; Observational studies; Experimental studies

\section{Introduction}

Atrial fibrillation (AF) is the most prevalent sustained cardiac arrhythmia in the world [1]. Its prevalence is almost twice as high as the observed in the past decade, but data about AF may

Manuscript submitted August 3, 2020, accepted August 18, 2020

Published online September 21, 2020

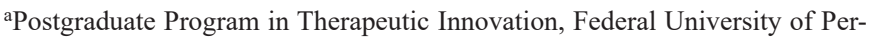
nambuco (PPGIT/UFPE), Recife, PE, Brazil

bPernambuco Cardiovascular Emergency Room Teacher Luiz Tavares (PROCAPE/UPE), Recife, PE, Brazil

${ }^{\mathrm{c}}$ Faculty of Medical Sciences, University of Pernambuco (FCM/UPE), Recife, PE, Brazil

${ }^{\mathrm{d}}$ Health and Biological Sciences Program, University of Vale do Sao Francisco (PPGCSB/UNIVASF), Petrolina, PE, Brazil

eAggeu Magalhaes Institute, Oswaldo Cruz Foundation (Fiocruz), Recife, PE, Brazil

${ }^{\mathrm{f} C}$ Corresponding Author: Maria Mariana Barros Melo da Silveira, Pernambuco Cardiovascular Emergency Room Teacher Luiz Tavares (PROCAPE/UPE), Palmares Street, Santo Amaro, Recife, PE 50100-060, Brazil.

Email: marianabms@gmail.com

doi: https://doi.org/10.14740/jocmr4313 be underestimated because the asymptomatic form is not diagnosed frequently [2]. AF is associated with a mortality increase of approximately two times that observed among patients with sinus rhythm [3].

$\mathrm{AF}$ genesis is related to electrophysiological alterations in the atrial tissue that foster and propagate the abnormal electric impulse. Many clinical conditions are considered risk factors for the development of $\mathrm{AF}$, and possibly influence in the prevalence rise observed in the last decades [2].

Atrial fibrosis is an induction factor of cardiac structural remodeling, which can create arrhythmogenic substrate for the emergence of AF $[4,5]$. Studies performed with humans and animals show that left atrial fibrosis is involved with this arrhythmia physiopathology [6-8]. Better understanding of the relation between atrial fibrosis and AF can allow for the identification of a higher-risk subgroup, and may have an impact in the management of those patients (rhythm control, frequency or ablation control) [6].

The exact mechanism leading to atrial fibrosis still remains undefined, however, some studies demonstrated that the activation of the immune system and inflammatory response play a role in the physiopathology of atrial fibrosis[1, 5]. In this scenario, galectin-3 (Gal-3) has been considered one of the possible substances contributing to cardiac fibrosis [5].

The role of Gal-3 in the pathogenesis of cardiac fibrosis involves macrophages and fibroblasts recruitment in the myocard, resulting in cell proliferation and collagen accumulation $[5,9,10]$, and it plays a role in the electric atrial activity and structural remodeling [1]. Studies showed that high circulating Gal-3 concentrations were associated with an increased risk of developing AF $[1,5,9,10]$. Despite of inferences that point to Gal-3 playing a role in the atrial fibrosis and $\mathrm{AF}$ physiopathology, there is still a need for further understanding of the molecular basis and clinical associations of it [11, 12] (Fig. 1).

Better understanding of Gal-3 association with atrial fibrosis in patients with AF will allow for progress in the understanding of this arrhythmia physiopathology and contribute for a more appropriate clinical management of carrier patients or those with high risk for the development of AF. The present study aimed at assessing the association of Gal-3 with atrial fibrosis in patients with AF. 


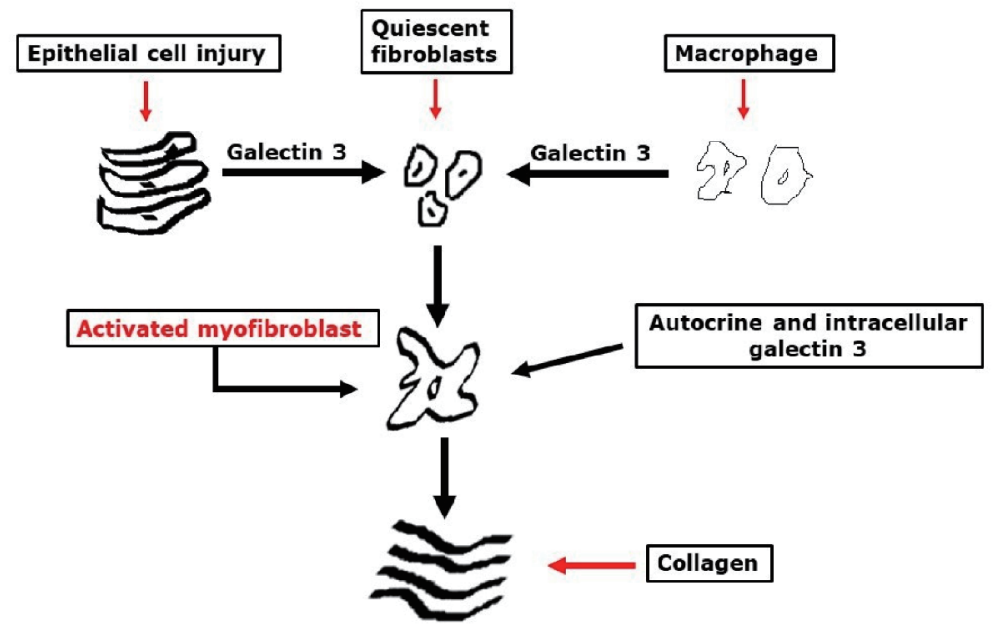

Figure 1. Potential mechanistic roles of galectin-3 in tissue fibrogenesis.

\section{Methods}

The present study is a systematic review of literature following the guidelines of Preferred Reporting Items for Systematic Reviews and Meta-Analyses (PRISMA) statement [13]. The question asked in this review was: "What is the relation between Gal-3 and atrial fibrosis in patients with AF?" Using PICOS [14] search strategy, we considered eligible studies in which the population was composed of patients with AF, where Gal-3 had been assessed as a possible marker for cardiac fibrosis and those studies that were clinical, observational or experimental studies.

The following databases were searched up until April 2020: Cochrane Library, British Medical Journal (BMJ) Best Practice, National Library of Medicine National Institutes of Health (PubMed/MEDLINE), World Wide Science, Science Direct, Literatura Latino-Americana e do Caribe em Ciencias da Saude (LILACS), and Directory of Open Access Journal e University of Alberta Library. Search strategy was guided by the use of controlled vocabulary according to Descritores em Ciencias da Saude (DeCs) as an extended translation of Medical Subject Headings (MeSH). In DeCs, the search was carried out in Portuguese and keywords were obtained in English, and their combination was done using the boolean operators "and" and "or". Keywords used in this study were: "galactin-3", "atrial fibrosis", "atrial fibrillation", "cardiovascular disease", "clinical studies", "observational studies", and "experimental studies".

The search for the studies was guided by the research question and the combination of the keywords in the database, aiming to find the term in the article title, abstract and keywords. There were no restrictions with language but articles from 2010 to 2020 were given priority. Excluded studies encompassed review articles, systematic reviews, meta-analysis and grey literature. Keywords were combined two by two in each database, and when necessary, we chose to use a triple combination.

First, titles and abstracts were read and each researcher registered the agreement or not to the inclusion of the study (the first step). Disagreeing cases were discussed and submitted to a third evaluation performed by a more experienced researcher (the second step). Second, selected articles were fully read aiming to find the answer to the research question of the review (the third step). Figure 2 describes the flow for the identification, selection, eligibility and inclusion of studies.

Data extraction was carried out independently by two researchers with the aid of an electronic form built using Microsoft Excel $^{\circledR}$, and a data compatibility assessment was performed by a third researcher, in order to evaluate consistency and cleanliness of data. Kappa coefficient was used to assess the inter-observer reliability. Critical review of studies' quality of data was carried out using Oxford Centre Evidence Based Medicine [15], as well as the methodology quality of studies was evaluated using GRADE [16] guidelines, using a check list with questions (Supplementary Material 1, www.jocmr. org) in order to assess the scientific inference and significance. Study data synthesis was guided by PRISMA, following the structure rationale IMRaD (Introduction, Methods, Results and Discussion).

\section{Results}

A total of 12 studies fulfilled the eligibility criteria established and their main information is listed in Table $1[5,10-$ $12,17-24]$. Inter-observer reliability of the study relevance was considered substantial (Kappa $=0.67)$, and it pointed to the homogeneity of the articles included in the study. As shown here (Supplementary Material 1, www.jocmr.org), with regard to the relevance of the outcomes presented, there was an agreement that $100 \%(n=12)$ of studies analyzed present scientific relevant results, as well as $100 \%$ of studies presented a clear methodology and in accordance to the main outcome.

Articles were analyzed with regard to quality and categorized by degree of recommendation and level of evidence [15]. It was observed that $91.66 \%(\mathrm{n}=11)$ of the articles were classi- 


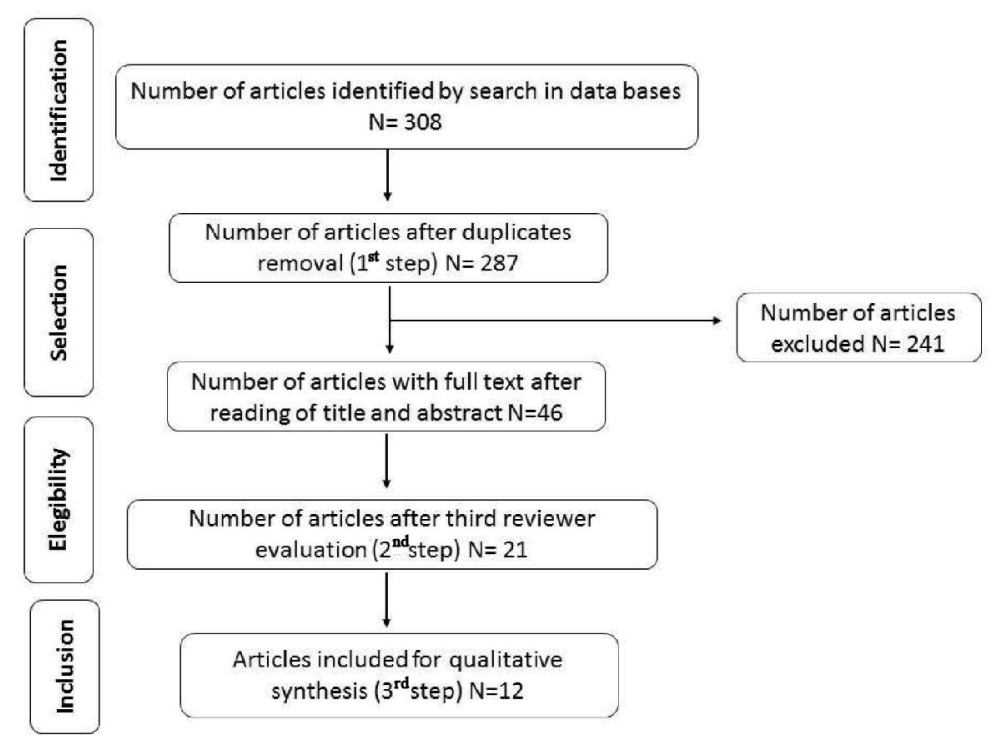

Figure 2. Results flowchart of studies search and selection strategy.

fied as grade $\mathrm{B}$ of recommendation (moderate), and one article $(8.34 \%)$ as grade A of recommendation (high). Cohort studies were the most frequent type $62.5 \%(\mathrm{n}=10)$. Case-control studies were $58.3 \%(n=7), 33.3 \%(n=4)$ were prospective cohort and $8.4 \%(n=1)$ were ecological studies (Table 1$)$.

\section{Discussion}

Twelve studies that fit the inclusion criteria were included in the present review [5, 10-12, 17-24], comprising a total of 9,470 individuals. Age of study subjects ranged from 46.1 to 70 years old. All studies demonstrated that Gal-3 levels are higher in patients with AF non-valvular and that it can be considered a marker for fibrosis in this population (Table 1).

Stanojevic et al (2019) [17] compared levels of Gal-3 in patients with acute myocardial infarction (AMI) and AF in order to investigate the role of Gal-3, and they found that patients with AMI and AF had higher levels of Gal-3 than those without AF $(\mathrm{P}<0.05)$, and that Gal-3 can be considered and independent predictive factor for the emergence of AF.

Fashanu et al (2017) [12] in the ARIC study, analyzed if Gal-3 was associated with AF incidence in a cohort of 8,436 individuals, and found out that subjects with elevated Gal-3 levels presented higher risk of developing AF $(\mathrm{P}<0.0001)$. The association of Gal-3 with AF incidence was very strong in the first year of follow-up, and it can be explained by the role Gal-3 plays in myocardial fibrosis. The association between plasma Gal-3 and AF was moderately strong and independent of baseline AF risk factors. This association was accounted for by participants with high Gal-3 levels having developed more heart failure (HF) during follow-up of AF. Gal-3 may increase AF risk via pathways involving HF [12]. Most articles excluded patients with HF.

In a study assessing whether Gal-3 was involved in the evolution and in the progress of coronary cardiac disease and in the progress of AF, Gal-3 was considered a biomarker of severity and/or atherosclerotic plaque instability. It was observed that elevated levels of Gal-3 are significantly associated with AF patients $(\mathrm{P}<0.05)$. Elevated levels of Gal-3 can stimulate macrophage migration, proliferation of fibroblasts and fibrosis development, being the last one as an important factor affecting the process of myocardial fibrosis [18]. When the type of AF was compared, levels of Gal-3 were significantly higher in patients with persistent AF than in those with paroxysmal AF $(\mathrm{P}<0.001)$.

Gurses et al (2015) [10] also observed that elevated levels of Gal-3 are associated with AF (P $<0.001)$, and it can be considered an independent $\mathrm{AF}$ predictor in a multivariate analysis $(\mathrm{P}<0.001)$. When the relation was assessed comparing the type of AF, levels of Gal-3 were significantly higher in patients with persistent AF than in those with paroxysmal AF $(\mathrm{P}<0.001)$. These results suggest that Gal-3 is involved in the structural remodeling process in those patients.

Berger and colleagues, in 2017 [19] aimed at assessing the levels of serum Gal-3 and in the left atrial tissue in patients to be submitted to thoracoscopic surgery for AF, and investigated if the baseline levels of Gal-3 or its alteration had any impact in the fibrotic process that contribute to the arrhythmogenic substrate after AF modification to the sinus rhythm. As a result, they observed that an increase in the levels of Gal-3 was significantly associated with a higher recurrence of AF after its correction by surgical procedure $(\mathrm{P}=0.014)$.

Clementy et al (2016) also verified that patients with an elevated level of Gal-3 presented more frequently AF recurrence after ablation $(\mathrm{P}=0.0006)$. In a univariate analysis, Gal-3 was an $\mathrm{AF}$ recurrence predictor $(\mathrm{P}=0.02)$, and in a multivariate analysis it was an independent predictor of new AF events $(\mathrm{P}=$ 0.02). Thus, they concluded that high levels of Gal-3 are strong predictors of AF recurrence after ablation, independently of its type [22]. As a limitation, Clementy et al (2016) [22] showed that patients with HF were also included in the study, despite 


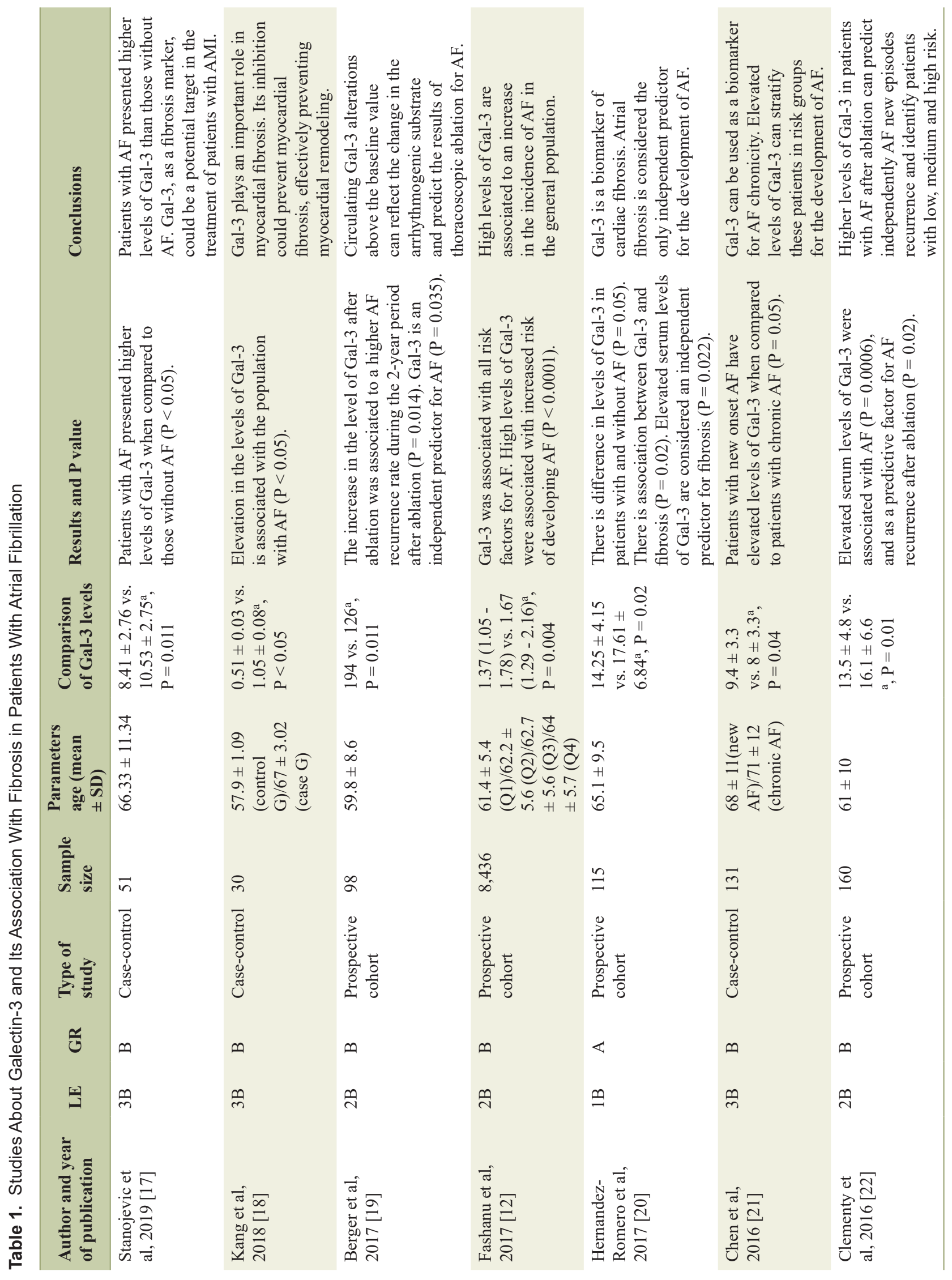




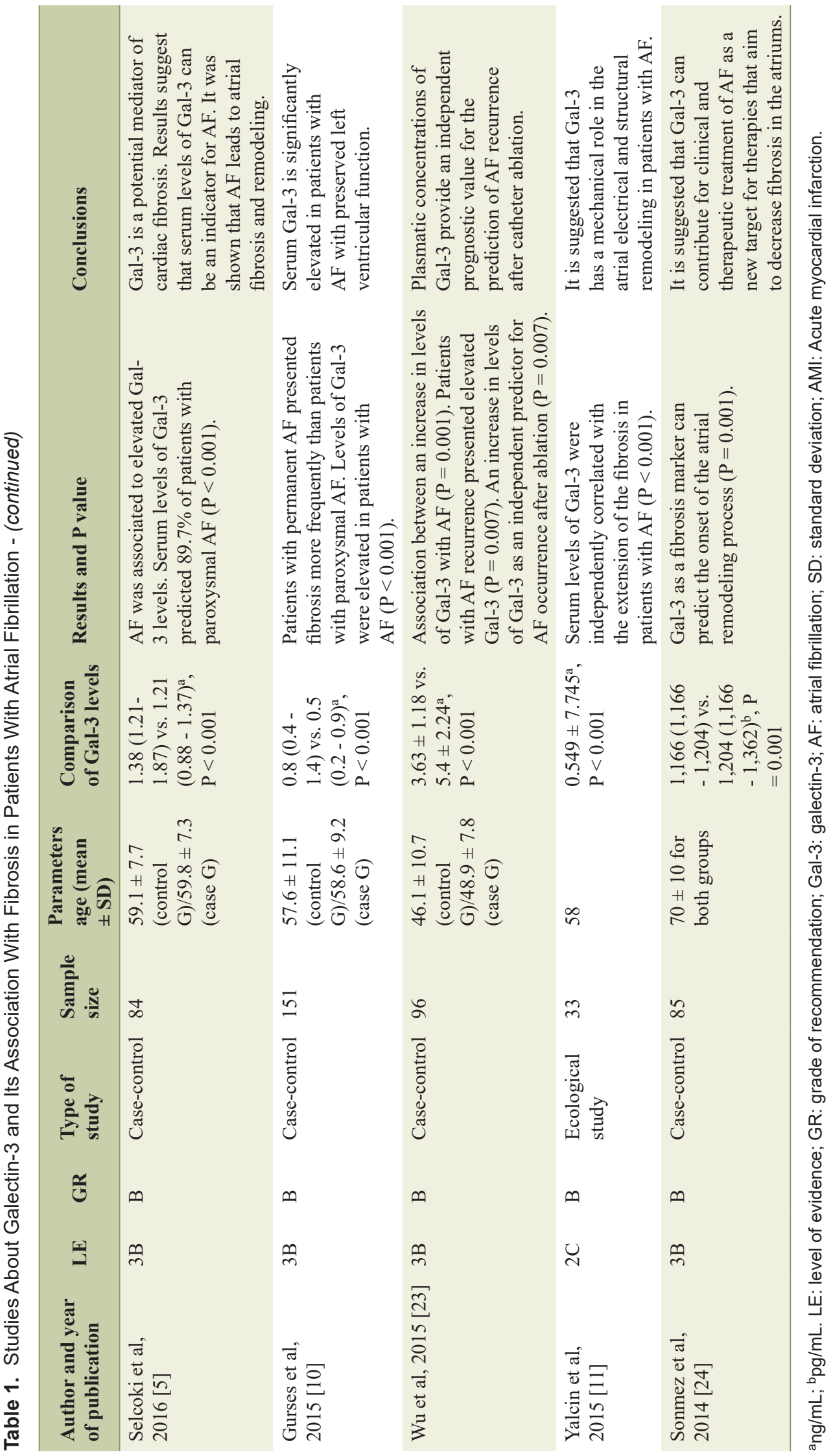


the bias that Gal-3 levels are greater in this population. In addition, Gal-3 remained an independent predictor of AF recurrence even after removing HF patients from the population.

Furthermore, the findings of Berger and colleagues suggest that the increase in the levels of Gal-3 is determined by the presence of AF, reflecting a process that can cause the disease. In those patients, the increase in the levels of Gal-3 occurs with time and it leads to a fibrotic process, increasing the risk of arrhythmogenic substrate development that remains or develops further, independently of AF modification to sinus rhythm. In a multivariate analysis, it was observed that Gal-3 is an independent $A F$ recurrence predictor $(P=0.035)$ [19]. As a conclusion of their study, Gal-3 is an independent variable for predicting risk in patients with $\mathrm{AF}$, playing an important role as a potential marker for structural remodeling. Fibrosis is an important component of the arrhythmogenic substrate, and patients with AF have an increase in the load of fibrosis [19].

In some studies, females presented higher levels of Gal-3 when compared to males $(\mathrm{P}<0.0001)[12],(\mathrm{P}=0.03)[21]$, $(\mathrm{P}$ $=0.046)$ [19], however, Stanojevic et al (2019) [17] and Selcoki et al (2016) [5] found out that males presented elevated levels of Gal-3 in univariate and multivariate analysis $(\mathrm{P}<0.001$ [17] and $\mathrm{P}=0.047$ [5], respectively). Black patients presented higher levels of Gal-3 when compared to white patients $(\mathrm{P}<$ $0.00001)$ [12].

Hernandez-Romero and colleagues (2017) evaluated the role of Gal-3 in atrial remodeling and they found out that there are differences in the increase of serum levels of Gal-3 between patients with and without $\operatorname{AF}(P=0.02)$; besides that, they found association between elevated levels of Gal-3 and fibrosis $(\mathrm{P}=0.015)$. Atrial remodeling evaluated as tissue atrial fibrosis remained an independent factor for AF development ( $\mathrm{P}$ $=0.022)$ [20].

Study results indicate that the pro-fibrotic effects of Gal3 and interstitial atrial remodeling are converging processes in fibrosis development and consequently in the emergence of AF. Atrial fibrosis is an independent predictor of post-operative AF occurrence $(\mathrm{P}=0.022)[20]$.

With the goal of determining if Gal-3 is related to atrial remodeling markers and fibrosis extension, the study carried out by Yalcin et al (2015) showed that the increase in Gal-3 is related to cardiac fibrosis in patients with $\mathrm{AF}(\mathrm{P}<0.001)$, and it is independently correlated to the fibrosis extension in the left atrium $(\mathrm{P}<0.001)$, suggesting a possible mechanical role of Gal-3 in atrial electrical and structural remodeling [11].

When estimating whether the extension of Gal-3 circulation varied between patients with new onset $\mathrm{AF}$ and chronic AF, Chen and colleagues (2016) found out that Gal-3 is independently related to new onset AF, that is, to the early stage of AF $(P=0.05)$. Thus, researchers suggest that Gal-3 can be a chronicity biomarker for AF, which could be used to ease the diagnosis of the early stage of the disease in high-risk patients, because elevated levels of Gal-3 can stratify the risk of developing AF [21].

Sonmez et al (2014), found significant results in patients with AF and elevation in the levels of Gal-3 ( $\mathrm{P}=0.001)$, suggesting that Gal-3 can be used as a new target to lower the level of atrial fibrosis and inflammation in patients with AF. In- creased Gal-3 leads to an increased number of monocytes and macrophages involved in cell interactions during atrial remodeling. Gal-3 and other fibrosis markers can predict the onset of the atrial remodeling process and contribute to the clinical and therapeutic treatment of AF, with a new target aiming at decreasing atrial fibrosis and inflammation [24].

Selcoki et al (2016) reported that serum levels of Gal-3 and left atrial diameter (LAD) were significantly elevated in patients with paroxysmal AF $(\mathrm{P}<0.001$ and $\mathrm{P}=0.02$, respectively), as well as the association of elevated levels of Gal-3 and an increase in the LAD $(\mathrm{P}<0.001)$. Elevated levels of Gal-3 predicted $89.7 \%$ of patients with paroxysmal AF $(\mathrm{P}<$ $0.001)$. These results strongly suggest that the levels of Gal-3 can be AF markers and can reflect structural remodeling of the left atrium in patients with paroxysmal AF [5].

$\mathrm{Wu}$ et al (2015) also verified that the concentration of Gal-3 in patients with AF was elevated $(\mathrm{P}=0.001)$. Patients with AF recurrence presented elevated circulating Gal-3 (P $=0.007)$, being an independent predictor for the occurrence of AF after arrhythmia circuit ablation by radiofrequency in a multivariate analysis $(\mathrm{p}=0.007)$, thus providing diagnostic value. Patients with persistent AF presented higher LAD (P $<0.001)$. LAD and Gal-3 were independent predictors for the occurrence of AF after ablation $(\mathrm{P}=0.02$ and $\mathrm{P}=0.03$, respectively). However, Gal-3 cannot be described as a cause or a consequence of AF [23].

Fibrosis is an important component of the AF arrhythmogenic substrate, and patients with the disease show an increase in the fibrosis. A study showed that the presence of thick fibrotic strands in atrial tissue can harness the re-entry activation and contribute to the AF arrhythmogenic substrate [25].

\section{Conclusions}

Studies demonstrated an association between elevated serum levels of Gal-3 and the presence of AF, either in the persistent or permanent paroxysmal form, or in the recurrence of this arrhythmia after the ablation procedure. This association can be explained by the interaction of Gal-3 with cardiac fibrosis when we consider that these patients are more likely to present atrial fibrosis.

\section{Supplementary Material}

Suppl 1. Quality Assessment of Study Methodology According to GRADE.

\section{Acknowledgments}

None to declare.

\section{Conflict of Interest}

The authors declare no conflict of interest. 


\section{Financial Disclosure}

This study was financed in part by the Coordenacao de Aperfeicoamento de Pessoal de Nivel Superior - Brasil (CAPES) - Finance Code 001.

\section{Author Contributions}

MMBMS: conceptualization, literature search, analysis, interpretation, writing first draft, reviewed and finalized the manuscript. JVBC: conceptualization, literature search, analysis, interpretation, reviewed and finalized the manuscript. BMS: literature search and analysis. LHPH: literature search and analysis. SLMA: literature search and analysis. ATX: conceptualization, literature search, analysis, interpretation, reviewed and finalized the manuscript. EBMF: conceptualization, literature search, analysis, interpretation, reviewed and finalized the manuscript. LRSV: conceptualization, administrative support, interpretation, reviewed and finalized the manuscript. DCSF: conceptualization, administrative support, interpretation, reviewed and finalized the manuscript. DCO: conceptualization, administrative support, interpretation, reviewed and finalized the manuscript.

\section{Data Availability}

The authors declare that data supporting the findings of this study are available within the article.

\section{References}

1. Pranata R, Yonas E, Chintya V, Tondas AE, Raharjo SB. Serum Galectin-3 level and recurrence of atrial fibrillation post-ablation - Systematic review and meta-analysis. Indian Pacing Electrophysiol J. 2020;20(2):64-69.

2. Magalhaes LP, Figueiredo MJO, Cintra FD, Saad EB, Kuniyoshi RR, Menezes Lorga Filho A, D'Avila ALB, et al. Executive summary of the II brazilian guidelines for atrial fibrillation. Arq Bras Cardiol. 2016;107(6):501508.

3. Shen H, Wang J, Min J, Xi W, Gao Y, Yin L, Yu Y, et al. Activation of TGF-beta1/alpha-SMA/Col I Profibrotic Pathway in Fibroblasts by Galectin-3 Contributes to Atrial Fibrosis in Experimental Models and Patients. Cell Physiol Biochem. 2018;47(2):851-863.

4. Nortamo S, Ukkola O, Lepojarvi S, Kentta T, Kiviniemi A, Junttila J, Huikuri H, et al. Association of sST2 and hsCRP levels with new-onset atrial fibrillation in coronary artery disease. Int J Cardiol. 2017;248:173-178.

5. Selcoki Y, Aydin HI, Celik TH, Isleyen A, Erayman A, Demircelik MB, Demirin H, et al. Galectin-3: A biochemical marker to detect paroxysmal atrial fibrillation? Clin Invest Med. 2016;39(6):27528.

6. Dunn MI, Marcum JL. Atrial mechanical performance following internal and external cardioversion of atrial fi- brillation: its relationship to peripheral embolization and acute cerebrovascular accident. Chest. 2002;121(1):1-3.

7. Marrouche NF, Wilber D, Hindricks G, Jais P, Akoum N, Marchlinski F, Kholmovski E, et al. Association of atrial tissue fibrosis identified by delayed enhancement MRI and atrial fibrillation catheter ablation: the DECAAF study. JAMA. 2014;311:498-506.

8. Smaill BH. Fibrosis, myofibroblasts, and atrial fibrillation. Circ Arrhythm Electrophysiol. 2015;8(2):256-257.

9. Ho JE, Yin X, Levy D, Vasan RS, Magnani JW, Ellinor PT, McManus DD, et al. Galectin 3 and incident atrial fibrillation in the community. Am Heart J. 2014;167(5):729-734 e721.

10. Gurses KM, Yalcin MU, Kocyigit D, Canpinar H, Evranos B, Yorgun H, Sahiner ML, et al. Effects of persistent atrial fibrillation on serum galectin-3 levels. Am J Cardiol. 2015;115(5):647-651.

11. Yalcin MU, Gurses KM, Kocyigit D, Canpinar H, Canpolat U, Evranos B, Yorgun H, et al. The association of serum galectin-3 levels with atrial electrical and structural remodeling. J Cardiovasc Electrophysiol. 2015;26(6):635640.

12. Fashanu OE, Norby FL, Aguilar D, Ballantyne CM, Hoogeveen RC, Chen LY, Soliman EZ, et al. Galectin-3 and incidence of atrial fibrillation: the Atherosclerosis Risk in Communities (ARIC) study. Am Heart J. 2017;192:1925.

13. Galvao TF, Pansani TS. Principais itens para relatar Revisoes sistematicas e Meta-analises: A recomendacao PRISMA. Epidemiol e Servicos Saude. 2015;24(2):335342.

14. Galvao TF, Pereira MG. Revisoes sistematicas da literatura: passos para sua elaboracao. Epidemiol e Servicos Saude. 2014;23(1):183-184.

15. OCEBM Levels of Evidence Working Group, Durieux N, Pasleau F, Howick J. The Oxford 2011 Levels of Evidence. Group [Internet]. 2011;1(version):5653. Available from: http://www.cebm.net/index.aspx? $\mathrm{o}=1025$.

16. Guyatt G, Oxman AD, Akl EA, Kunz R, Vist G, Brozek J, Norris S, et al. GRADE guidelines: 1. IntroductionGRADE evidence profiles and summary of findings tables. J Clin Epidemiol. 2011;64(4):383-394.

17. Stanojevic D, Apostolovic S, Stokanovic D, Momcilovic S, Jevtovic-Stoimenov T, Salinger-Martinovic S, Kostic $\mathrm{T}$, et al. Galectin-3 in acute myocardial infarction patients with atrial fibrillation. Med Princ Pract. 2019;28(3):284290.

18. Kang Q, Li X, Yang M, Fernando T, Wan Z. Galectin-3 in patients with coronary heart disease and atrial fibrillation. Clin Chim Acta. 2018;478:166-170.

19. Berger WR, Jagu B, van den Berg NWE, Chan Pin Yin D, van Straalen JP, de Boer OJ, Driessen AHG, et al. The change in circulating galectin-3 predicts absence of atrial fibrillation after thoracoscopic surgical ablation. Europace. 2018;20(5):764-771.

20. Hernandez-Romero D, Vilchez JA, Lahoz A, RomeroAniorte AI, Jover E, Garcia-Alberola A, Jara-Rubio R, et al. Galectin-3 as a marker of interstitial atrial remodelling involved in atrial fibrillation. Sci Rep. 2017;7:40378. 
21. Chen D, Procter N, Goh V, Liu S, Chua SJ, Assadi-Khansari B, Stewart S, et al. New onset atrial fibrillation is associated with elevated galectin-3 levels. Int J Cardiol. 2016;223:48-49.

22. Clementy N, Benhenda N, Piver E, Pierre B, Bernard A, Fauchier L, Pages JC, et al. Serum galectin-3 levels predict recurrences after ablation of atrial fibrillation. Sci Rep. 2016;6:34357.

23. Wu XY, Li SN, Wen SN, Nie JG, Deng WN, Bai R, Liu N, et al. Plasma galectin-3 predicts clinical outcomes after catheter ablation in persistent atrial fibrillation patients without structural heart disease. Europace.
2015;17(10):1541-1547.

24. Sonmez O, Ertem FU, Vatankulu MA, Erdogan E, Tasal A, Kucukbuzcu S, Goktekin O. Novel fibro-inflammation markers in assessing left atrial remodeling in nonvalvular atrial fibrillation. Med Sci Monit. 2014;20:463470.

25. Krul SP, Berger WR, Smit NW, van Amersfoorth SC, Driessen AH, van Boven WJ, Fiolet JW, et al. Atrial fibrosis and conduction slowing in the left atrial appendage of patients undergoing thoracoscopic surgical pulmonary vein isolation for atrial fibrillation. Circ Arrhythm Electrophysiol. 2015;8(2):288-295. 